\title{
Fluxo assistencial de usuárias com câncer de mama na rede pública de atenção à saúde*
}

Silvia Rosa de Souza Tolêdo ${ }^{1}$, Nilza Alves Marques Almeida², Marta Rovery de Souza ${ }^{3}$, Ruth Minamisava ${ }^{4}$, Ruffo Freitas Júnior ${ }^{5}$

\footnotetext{
* Artigo baseado na dissertação

"Prevalência e Fatores Associados ao Fluxo Assistencial de Usuárias com Câncer de Mama na Rede Pública de Atenção à Saúde em Goiânia, Goiás" apresentada ao Programa de Pós-Graduação em Saúde Coletiva da Universidade Federal de Goiás, em 2012.

${ }^{1}$ Enfermeira, Mestre em Saúde Coletiva. Professora Assistente da Pontifícia Universidade Católica de Goiás. Goiânia, GO, Brasil. E-mail:

silviarosatoledo@gmail.com.

${ }^{2}$ Enfermeira, Doutora em Ciências da Saúde. Professora Adjunto da Universidade Federal de Goiás. Goiânia, GO, Brasil. Email: nilzafenufg@gmail.com.

${ }^{3}$ Bacharel em Ciências Sociais, Doutora em Ciência Sociais. Professora Associada da Universidade Federal de Goiás. Goiânia, GO, Brasil. E-mail: martary@gmail.com.

${ }^{4}$ Enfermeira, Doutora em Medicina Tropical. Professora Associada da Universidade Federal de Goiás. Goiânia, GO, Brasil. E-mail:

minamisava@gmail.com.

${ }^{5}$ Médico, Doutor em Tocoginecologia. Professor Adjunto da Universidade Federal de Goiás. Goiânia, GO, Brasil. E-mail. Goiânia, GO, Brasil. E-mail: ruffojr@terra.com.br.
}

Recebido: 23/12/2015.

Aceito: 29/09/2016.

Publicado: 22/12/2016.

Como citar esse artigo:

Tolêdo SRS, Almeida NAM, Souza MR, Minamisava R, Freitas Júnior R. Fluxo assistencial de usuárias com câncer de mama na rede pública de atenção à saúde. Rev. Eletr. Enf. [Internet]. 2016 [acesso em: ____18:e1201. Disponível em: http://dx.doi.org/10.5216/ree.v18.39147.

\section{RESUMO}

Estudo transversal analítico que objetivou analisar a prevalência e os fatores associados ao fluxo assistencial nos três níveis de atenção à saúde exclusivamente no Sistema Único de Saúde (SUS) de usuárias com câncer de mama. Entrevistaram-se 92 mulheres com câncer de mama, entre 29 e 82 anos, em tratamento ambulatorial em nível terciário de atenção à saúde do SUS. A prevalência de utilização do fluxo realizado exclusivamente no SUS, nos três níveis de atenção à saúde, foi de 43,5\% (IC95\% 33,6-53,7). Cerca de um terço das mulheres realizou consultas ou exames na rede privada, principalmente a mamografia. Os fatores associados à utilização do fluxo exclusivamente SUS, nos três níveis, foi idade $\leq 20$ anos na primeira consulta ginecológica $(O R=2,55)$ e estar sem trabalho remunerado $(O R=3,28)$. Estes resultados sugerem a necessidade de melhoria de acesso na rede pública de atenção à saúde para mulheres com câncer de mama.

Descritores: Neoplasias da Mama; Acesso aos Serviços de Saúde; Serviços de Saúde da Mulher.

\section{INTRODUÇÃO}

O sistema de saúde brasileiro é composto pelos subsistemas público e privado. O subsistema público, representado pelo Sistema Único de Saúde (SUS), tem financiamento e gestão integrada dos governos federal, estaduais e municipais, incluindo os serviços privados contratados e/ou conveniados. O subsistema privado de saúde é dividido nos subsetores de saúde suplementar e liberal clássico 
(serviços particulares autônomos). O subsetor de saúde suplementar é composto por prestadores de serviços próprios, diferentes tipos de planos privados de saúde e por serviços financiados com recursos públicos ou privados, por apólices de seguro, além de conter subsídios fiscais. Os componentes público e privado do sistema, embora distintos, estão interconectados ${ }^{(1)}$. A organização da assistência à saúde no SUS foi concebida como uma rede articulada entre atenção primária, secundária e terciária, por níveis de complexidade. Os serviços de atenção básica, responsáveis pelos serviços primários de saúde, são descentralizados e se localizam próximos da população e os serviços de atenção secundária e terciária são regionalizados. O SUS possui sua rede de serviços de atenção primária, mas tem poucos serviços próprios na atenção secundária, que são predominantemente oferecidos pelo subsetor de saúde suplementar contratado pelo SUS ${ }^{(1-2)}$.

A desarticulação entre a oferta e as demandas dos serviços resulta em iniquidades no acesso e em ineficiência do sistema de saúde. A frágil regulação do acesso e o fluxo desordenado de usuários levam à baixa resolubilidade dos casos atendidos. Por isso, o fluxo assistencial do SUS visa otimizar a trajetória dos usuários para alcançar a resolubilidade, facilitar o acesso e contribuir para a integralidade e a equidade em saúde ${ }^{(2)}$. Deve iniciar, preferencialmente, pela atenção básica, que desempenha o papel ordenador e coordenador dos fluxos e contrafluxos de seus usuários. Entretanto, os mecanismos regulatórios do SUS ainda estão sendo estruturados e não são robustos nem eficientes ${ }^{(1-2)}$.

Indivíduos com doenças crônico-degenerativas utilizam com frequência os fluxos assistenciais, dada à necessidade de maior número de consultas e de exames de controle. Dentre as doenças degenerativas, o câncer de mama é o mais importante em mulheres no Brasil e no mundo, excluído o câncer de pele não melanoma ${ }^{(3-4)}$. Para a população sintomática, o Ministério da Saúde recomenda o fluxo assistencial a partir da atenção primária que deve identificar os casos suspeitos, priorizar o encaminhamento ao nível secundário para avaliação por imagem e confirmação diagnóstica. Nos casos confirmados, o tratamento é realizado nos serviços de atenção terciária ${ }^{(5-6)}$. Esse fluxo deve apresentar um contínuo de ações interligadas e integradas de linhas de cuidado para favorecer a assistência adequada ${ }^{(7-8)}$.

No Brasil, os casos de câncer de mama geralmente são diagnosticados tardiamente e/ou apresentam atraso no início do tratamento ${ }^{(9-10)}$. Também se observa no país, desigualdades na tendência da mortalidade por câncer de mama, com declínio nos estados de nível socioeconômico mais elevado e aumento nos estados mais pobres ${ }^{(11)}$. O padrão de utilização do SUS pode variar de acordo com fatores como o tipo de agravo à saúde e área geográfica. As desigualdades da atenção à saúde das mulheres com câncer de mama no país indicam a necessidade de estudos da integralidade da atenção à saúde oferecida pelo SUS.

A análise dos fluxos assistenciais no âmbito do SUS utilizados pelas mulheres com câncer de mama é uma forma de investigar nós críticos da integralidade da atenção à saúde. Assim, o objetivo deste estudo foi analisar a prevalência e os fatores associados ao fluxo assistencial nos três níveis de atenção à saúde exclusivamente no SUS, de usuárias com câncer de mama. 


\section{METODOLOGIA}

Estudo transversal analítico realizado em uma capital do Centro-Oeste Brasileiro. O estudo foi conduzido no ambulatório de Mastologia do Hospital das Clínicas da Universidade Federal de Goiás que é um hospital universitário, do nível terciário de atenção à saúde, da rede própria do SUS, do município de Goiânia/Goiás/Brasil. O presente estudo foi aprovado pelo Comitê de Ética em Pesquisa do Hospital das Clínicas da Universidade Federal de Goiás, Protocolo no 162/2011 e conduzido em conformidade com as normas brasileiras para pesquisas com seres humanos.

Foram incluídas no estudo, usuárias do ambulatório de Mastologia, maiores de 18 anos, residentes no município de Goiânia e em tratamento ambulatorial para o câncer de mama no ano de 2012. Foram excluídas do estudo as mulheres debilitadas fisicamente, com dificuldade para responder as perguntas da entrevista. A amostra foi de conveniência, com todas as usuárias presentes no serviço no período de 10 de fevereiro a 20 de maio de 2012. Todas as participantes foram entrevistadas pela pesquisadora em ambiente reservado, por meio de formulário estruturado, mediante assinatura do Termo de Consentimento Livre e Esclarecido.

Os fluxos assistenciais foram classificados em:

A. Fluxo assistencial nos três níveis de atenção à saúde exclusivamente no SUS;

B. Fluxo assistencial nos níveis primário e terciário no SUS;

C. Fluxo assistencial em campanha de rastreamento e no nível terciário no SUS;

D. Fluxo assistencial nos níveis primário e terciário no SUS e, fluxo assistencial no subsistema privado no nível secundário;

E. Fluxo assistencial no nível secundário no subsistema privado e fluxo assistencial no nível terciário no SUS. As prevalências dos diferentes fluxos assistenciais foram calculados com seus respectivos intervalos de $95 \%$ de confiança usando o software OpenEpi (http://openepi.com/OE2.3/Menu/OpenEpiMenu.htm).

Foram considerados como nível de atenção primária, campanha de rastreamento e consulta médica não especializada. Foram considerados como nível de atenção secundária os exames de imagem e as consultas especializadas.

Para a análise dos fatores associados ao desfecho fluxo assistencial nos três níveis de atenção à saúde exclusivamente no SUS, utilizou-se desfecho dicotomizado (sim/não). As variáveis de exposição foram: idade em anos; escolaridade em anos; casada (sim/não); trabalho remunerado (sim/não); casa própria (sim/não);

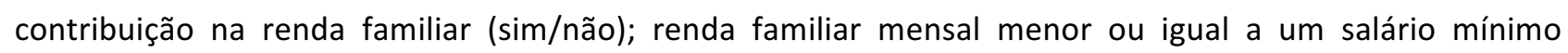
(sim/não); história familiar de câncer de mama (sim/não); idade na $1^{\text {a }}$ consulta ginecológica <20 anos (sim/não). A medida de associação das variáveis de exposição com a variável de desfecho foi o odds ratio $(\mathrm{OR})$, com uso do teste do qui-quadrado ou Fisher, quando apropriado, para análise bivariada. A análise multivariada foi feita por meio de regressão logística (backward stepwise). Variáveis que mostraram associação com desfecho com $p<0,10$ em análise bivariada foram incluídas no modelo multivariado e 0 
modelo final foi avaliado pelo teste de Hosmer e Lemeshow. Foram consideradas variáveis independentes associadas com desfecho aquelas que, após ajuste, apresentaram valor de $p<0,05$.

Foi realizada a comparação do SUS e do subsistema privado de saúde na proporção dos intervalos de tempo gasto pelas mulheres nas etapas de atendimento (da solicitação de agendamento da mamografia e da ultrassonografia até o retorno ao médico). Considerou-se como tempo satisfatório o intervalo $\leq 15$ dias para o acesso a cada uma das quatro etapas de atendimento: da solicitação até o agendamento do exame, do agendamento até a realização do exame, da realização até o acesso ao resultado do exame e, do acesso ao resultado do exame até o retorno ao médico. O intervalo de tempo de no máximo 15 dias foi definido baseado na recomendação do Ministério da Saúde de limite máximo de 60 dias entre o diagnóstico e início do tratamento do câncer de mama ${ }^{(12)}$. As comparações de proporções foram feitas utilizando o teste de quiquadrado.

As facilidades em utilizar um fluxo assistencial foram descritas, considerando as seguintes variáveis: agendamento da consulta especializada, consulta especializada, agendamento do início do tratamento e realização do tratamento no hospital. Todas essas variáveis foram dicotômicas (fácil, sim/não).

Os dados foram digitados no programa Microsoft Excel 2003 A análise dos dados foi realizada no programa SPSS versão 15.0 .

\section{RESULTADOS}

O estudo incluiu 92 mulheres em tratamento ambulatorial por câncer de mama com idade média de 56,1 anos (desvio-padrão=10,9 anos). A prevalência de mulheres em tratamento do câncer de mama que não utilizaram o subsistema privado de saúde foi de 67,4\% (n=62; IC95\% 57,3-76,4). A Tabela 1 mostra que menos da metade das mulheres (43,5\%; IC95\% 33,6-53,7) relataram fluxo assistencial nos três níveis de atenção à saúde exclusivamente no SUS (fluxo A). Quase 25\% das mulheres percorreram os fluxos assistenciais B e C e representam a proporção de mulheres com provável diagnóstico tardio, com encaminhamento imediato ao tratamento em nível terciário de atenção. Os fluxos assistenciais $D$ e $E$ mostram que $32,6 \%$ das mulheres estudadas utilizaram o subsistema privado no nível secundário de atenção à saúde.

Tabela 1: Tipos e prevalência dos fluxos assistenciais utilizados pelas 92 mulheres em tratamento ambulatorial por câncer de mama. Goiânia, GO, Brasil, 2012.

\begin{tabular}{|c|c|c|c|c|c|c|c|}
\hline \multirow{3}{*}{ Tipos de Fluxo Assistencial } & \multicolumn{4}{|c|}{ Níveis de atenção à saúde } & \multirow{3}{*}{$\mathbf{n}$} & \multirow{3}{*}{ Prevalência (\%) } & \multirow{3}{*}{ IC95\% } \\
\hline & \multicolumn{2}{|r|}{ Primário } & \multirow{2}{*}{ Secundário } & \multirow{2}{*}{ Terciário } & & & \\
\hline & Campanha & Consulta não especializada & & & & & \\
\hline$A$ & - & SUS & SUS & SUS & 40 & 43,5 & $33,6-53,7$ \\
\hline B & - & SUS & - & SUS & 9 & 9,8 & $4,9-17,2$ \\
\hline C & SUS & - & - & SUS & 13 & 14,1 & $8,1-22,4$ \\
\hline $\mathrm{D}$ & - & SUS & Privado & SUS & 12 & 13,0 & $7,3-21,1$ \\
\hline $\mathrm{E}$ & - & - & Privado & SUS & 18 & 19,6 & $12,4-28,6$ \\
\hline
\end{tabular}

Os potenciais fatores associados ao fluxo assistencial nos três níveis de atenção à saúde 
exclusivamente no SUS foram mulheres que realizaram a consulta ginecológica com idade $<20$ anos e as que não tinham trabalho remunerado (Tabela 2).

Tabela 2: Análise bivariada dos potenciais fatores associados ao fluxo assistencial nos três níveis de atenção à saúde exclusivamente no SUS, utilizado pelas mulheres para o tratamento do câncer de mama. Goiânia, GO, Brasil, 2012.

\begin{tabular}{|c|c|c|c|c|c|}
\hline \multirow{4}{*}{ Variáveis de exposição } & \multirow{2}{*}{\multicolumn{2}{|c|}{$\begin{array}{l}\text { Fluxo assistencial nos três níveis de atenção à saúde } \\
\text { exclusivamente no SUS }\end{array}$}} & \multirow{4}{*}{ OR* } & \multirow{4}{*}{$\begin{array}{c}\text { IC } \\
95 \% * *\end{array}$} & \multirow{4}{*}{$\begin{array}{l}\text { Valor de } \\
\qquad p\end{array}$} \\
\hline & & & & & \\
\hline & Sim & Não & & & \\
\hline & n/total (\%) & n/total (\%) & & & \\
\hline $\begin{array}{l}\text { Idade na } 1^{\text {a }} \text { consulta ginecológica } \\
\qquad 20 \text { anos }\end{array}$ & $20 / 40(50,0)$ & $16 / 52(30,8)$ & 2,25 & $\begin{array}{l}0,96- \\
5,29\end{array}$ & 0,061 \\
\hline $\begin{array}{l}\text { Escolaridade até ensino } \\
\text { fundamental }\end{array}$ & $28 / 40(70,0)$ & $30 / 52(57,7)$ & 1,71 & $\begin{array}{l}0,72- \\
4,09\end{array}$ & 0,225 \\
\hline Sem trabalho remunerado & $31 / 40(77,5)$ & $28 / 52(53,8)$ & 2,95 & $\begin{array}{l}1,18- \\
7,42\end{array}$ & 0,019 \\
\hline Casada & $18 / 40(45,0)$ & $26 / 52(50,0)$ & 0,82 & $\begin{array}{l}0,36- \\
1,87\end{array}$ & 0,634 \\
\hline Não contribui com a renda familiar & $15 / 40(37,5)$ & $15 / 52(28,8)$ & 1,48 & $\begin{array}{l}0,62- \\
3,56\end{array}$ & 0,380 \\
\hline $\begin{array}{l}\text { Renda familiar mensal } \leq 1 \text { salário } \\
\text { mínimo }\end{array}$ & $14 / 40(35,0)$ & $18 / 51(35,3)$ & 0,99 & $\begin{array}{l}0,42- \\
2,35\end{array}$ & 0,977 \\
\hline Não tem casa própria & $17 / 40(42,5)$ & $21 / 52(40,4)$ & 1,09 & $\begin{array}{l}0,47- \\
2,52\end{array}$ & 0,838 \\
\hline $\begin{array}{l}\text { História familiar de câncer de } \\
\text { mama }\end{array}$ & $3 / 40(7,5)$ & $9 / 52(17,3)$ & 0,39 & $\begin{array}{l}0,10- \\
1,54\end{array}$ & 0,219 \\
\hline
\end{tabular}

$* *$ IC $=$ Intervalo de confiança de $95 \%$

Os fatores independentemente associados ao fluxo assistencial nos três níveis de atenção à saúde exclusivamente no SUS foram consulta ginecológica com $<20$ anos de idade e sem trabalho remunerado (Tabela 3).

Tabela 3: Análise multivariada dos fatores associados ao fluxo assistencial nos três níveis de atenção à saúde exclusivamente no SUS relatados por mulheres com câncer de mama. Goiânia, GO, Brasil, 2012.

\begin{tabular}{cccc}
\hline Fatores Associados & OR $^{*}$ & IC 95,0\%** & Valor de $\mathbf{p}$ \\
\hline Idade na $1^{\text {a }}$ consulta ginecológica $<20$ anos & 2,55 & $1,04-6,26$ & 0,042 \\
Sem trabalho remunerado & 3,28 & $1,26-8,53$ & 0,015 \\
\hline
\end{tabular}

* OR $=$ odds ratio ajustado por idade

** IC $=$ Intervalo de confiança de $95 \%$

Em relação à realização de exames de mamografia $(n=80)$ e ultrassonografia $(n=62)$, a Tabela 4 mostrou que a maioria das entrevistadas gastou o tempo $\leq 15$ dias em cada etapa para realização desses exames. Proporcionalmente, o subsistema privado foi mais ágil que a rede pública de atenção à saúde para a realização da mamografia quando comparado à ultrassonografia. 
Tabela 4: Proporção de realização de mamografia $(n=80)$ e ultrassonografia $(n=62)$ pelo SUS e etapas desde a solicitação até o retorno ao médico. Goiânia, GO, Brasil, 2012.

\begin{tabular}{|c|c|c|c|}
\hline \multirow{2}{*}{ Etapas de atendimento com tempo gasto $\leq 15$ dias } & \multicolumn{2}{|c|}{$\%$ de mulheres } & \multirow{2}{*}{ Valor de $p$} \\
\hline & SUS & Não SUS & \\
\hline \multicolumn{4}{|l|}{ Mamografia } \\
\hline Da solicitação até o agendamento & 85,0 & 68,4 & 0,081 \\
\hline Do agendamento até a realização & 76,2 & 76,8 & 0,470 \\
\hline Da realização até o acesso ao resultado & 64,9 & 90,5 & 0,001 \\
\hline Do acesso ao resultado até o retorno ao médico & 62,5 & 95,5 & 0,001 \\
\hline \multicolumn{4}{|l|}{ Ultrassonografia } \\
\hline Da solicitação até o agendamento & 85,0 & 90,0 & 0,637 \\
\hline Do agendamento até a realização & 85,0 & 90,0 & 0,637 \\
\hline Da realização até o acesso ao resultado & 83,3 & 95,0 & 0,229 \\
\hline Do acesso ao resultado até o retorno ao médico & 67,5 & 90,5 & 0,051 \\
\hline
\end{tabular}

As principais facilidades de acesso relatadas pelas mulheres do fluxo assistencial nos três níveis de atenção à saúde exclusivamente no SUS foram: agendamento da consulta especializada (80\%), agendamento do início do tratamento (85\%) e realização do tratamento no hospital $(92,5 \%)$. As mulheres que percorreram o Fluxo E relataram mais facilidade para agendar $(82,4 \%)$ e realizar a consulta especializada (100\%) no subsistema privado do que realizar o tratamento no hospital $(72,2 \%)$. Houve maior proporção de mulheres que apontaram a consulta especializada na rede privada $(100 \%)$ como facilitadora do fluxo comparativamente às mulheres que realizaram consulta especializada no SUS (71,8\%), apresentando diferença significativa $(p=0,01)$.

\section{DISCUSSÃO}

Embora todas as participantes deste estudo sejam usuárias do SUS, menos da metade seguiu o fluxo assistencial nos três níveis de atenção à saúde exclusivamente no SUS (Fluxo A). Explicações para a baixa prevalência encontrada no Fluxo A dificilmente excluem a dificuldade no acesso aos serviços do SUS, particularmente o acesso aos serviços de diagnóstico precoce e pronto tratamento. Esta dificuldade no acesso ao diagnóstico precoce do câncer de mama pode ser atribuída a frágil estruturação da rede SUS de assistência, que se verifica por meio da sobrecarga dos centros especializados e da busca pelo serviço privado para se obter agilidade do diagnóstico e tratamento ${ }^{(10)}$. Por outro lado, penaliza as populações mais carentes, sem acesso aos serviços privados e/ou de saúde suplementar, provavelmente aumentando o diagnóstico tardio. Melhorar o acesso no nível secundário de atenção à saúde do SUS implica em garantir recursos humanos e financeiros, equipamentos e insumos.

A variação de acesso aos diferentes níveis de atenção à saúde também pode ser decorrente de carência de informações das mulheres sobre a funcionalidade do complexo subsetor público de saúde ${ }^{(13-14)}$. Mulheres que realizaram a primeira consulta ginecológica antes dos 20 anos de idade provavelmente tiveram acesso por disporem de informações sobre os serviços disponíveis no SUS. Verificou-se também maior chance de utilização do fluxo exclusivamente no SUS, nos três níveis de atenção à saúde, pelas mulheres sem trabalho remunerado. Essas mulheres provavelmente têm melhor condição para agendar e comparecer aos 
atendimentos, já que os serviços de atenção ambulatorial funcionam em horário comercial, o que não favorece o acesso à maioria dos usuários que trabalham ${ }^{(14-15)}$.

Com a ampliação da cobertura da atenção primária pelo SUS, há um aumento da pressão de demanda pelos serviços de nível secundário e terciário, gerando "nós" críticos como a dificuldade de acesso aos serviços diagnósticos. Em Goiânia, a cobertura dos serviços primários de saúde ficou em torno de $60 \%$ em 2012, com estimativa para $65,44 \%$ até $2017^{(16)}$. Todavia, faltam análises da oferta de serviços no nível secundário de atenção ao câncer de mama.

O fluxo assistencial D (SUS-privado-SUS), que foi seguido por 13\% das mulheres, sugere que a suspeita diagnóstica de câncer de mama ocorreu em Unidades Básicas de Saúde, assim como para as mulheres do fluxo A e B (43,5\% e 9,8\% das mulheres, respectivamente). Dentre as hipóteses que podem ser aventadas sobre o fluxo $D$, acredita-se que essas mulheres utilizaram planos de saúde ou recursos próprios com a intenção de agilizar o diagnóstico ${ }^{(8)}$.

Cerca de $20 \%$ das mulheres que seguiram o fluxo E (privado-SUS), certamente contavam com plano de saúde ou recursos próprios apenas para atendimento no nível secundário. De fato, sabe-se que parte da população com nível socioeconômico mais baixo, usuária de plano de saúde de custo mais acessível, utiliza serviços e procedimentos de alto custo do SUS ${ }^{(1)}$. Nos últimos anos, têm sido apontada que esta é uma das consequências dos mecanismos de controle das seguradoras ${ }^{(17)}$. Porém, alguns estudos já indicam crescimento dos gastos dos planos privados na atenção de alta complexidade ${ }^{(18-19)}$ e que os recursos assistenciais pelo SUS são insuficientes para atender essa demanda ${ }^{(2)}$.

É possível que mulheres que seguiram os fluxos assistenciais B e $C$ tiveram diagnóstico tardio, porque houve encaminhamento direto da atenção primária para a terciária do SUS. No fluxo B as mulheres podem não ter sido identificadas oportunamente ou serem portadoras de câncer agressivo, não captado no rastreamento de rotina. No Brasil, as proporções de cobertura de rastreamento anual de câncer de mama são variadas, fato que potencialmente reduz as chances de sobrevida dessas mulheres ${ }^{(20-21)}$. Aproximadamente um terço dos tumores de mama em Goiânia é diagnosticado tardiamente ${ }^{(13)}$. A utilização do fluxo assistencial SUS somente no nível terciário (fluxo C) ocorreu por meio de campanhas de rastreamento. Embora o presente estudo não tenha sido desenhado para este fim, os resultados sugerem que as campanhas de rastreamento de câncer de mama, em Goiânia, contribuíram para a instituição do tratamento.

A maioria das mulheres incluídas no estudo referiu agilidade de atendimento para agendamento e atendimento nos serviços diagnósticos, mas as mulheres que usaram recursos próprios ou planos de saúde suplementar referiram maior agilidade na realização de mamografias. De fato, verificou-se que a mamografia realizada por meio dos planos de saúde suplementar ou recursos próprios foi realizada em menor tempo que no SUS. A desigualdade de acesso aos serviços diagnósticos está relacionada a baixas taxas de sobrevivência ${ }^{(13-14)}$ e evidencia fragilidades no alcance da resolubilidade da atenção à saúde.

Este é o primeiro estudo sobre os tipos de fluxo assistencial em Goiânia-GO, que identificou as 
características das pessoas que usam exclusivamente o SUS, com ênfase na abordagem da integralidade do cuidado às usuárias da rede de atenção à saúde do SUS. Uma limitação do presente estudo refere-se a possível superestimativa de prevalência das mulheres que estão seguindo o fluxo assistencial nos três níveis de atenção à saúde exclusivamente no SUS, pois não compuseram a amostra de estudo aquelas em tratamento ambulatorial no subsetor privado. Outra limitação se refere à amostra de conveniência e ao tamanho da amostra, sugerindo que as inferências dos resultados do presente estudo devem ser feitas com cautela.

\section{CONCLUSÕES}

O presente estudo mostrou diferentes fluxos assistenciais utilizados pelas mulheres com câncer de mama em Goiânia, Goiás. Menos da metade das mulheres utilizaram o fluxo assistencial nos três níveis de atenção à saúde exclusivamente no SUS. A principal dificuldade de acesso das usuárias da rede pública de atenção à saúde foi aos serviços de diagnóstico. Os fatores associados à utilização do fluxo exclusivamente SUS foi idade menor que 20 anos na primeira consulta ginecológica e estar sem trabalho remunerado.

As fragilidades na integralidade e na resolubilidade da atenção à saúde de portadoras de câncer de mama na rede SUS detectadas no presente estudo apontam desafios para as políticas públicas e para a regulação dos subsetores público e privado. Também indicam que são necessários futuros estudos para monitorar o acesso das mulheres com câncer de mama na rede pública de atenção à saúde.

\section{REFERÊNCIAS}

1. Paim J, Travassos C, Almeida C, Bahia L, Macinko J. The Brazilian health system: history, advances, and challenges. The Lancet [Internet]. 2011[acesso em: 10 nov 2015]; 377(9779):1778-1797. Disponível em: http://dx.doi.org/10.1016/S0140-6736(11)60054-8.

2. Ministério da Saúde. Implantação das Redes de Atenção à Saúde e outras estratégias da SAS [Internet] Brasília: Ministério da Saúde; 2014 [acesso em: 24 ago 2016]. Disponível em:

http://bvsms.saude.gov.br/bvs/publicacoes/implantacao_redes_atencao_saude_sas.pdf.

3. Facina T. Estimativa 2014: Incidência de câncer no Brasil. Rev Bras de Cancerologia. [Internet]. 2014 [acesso em: 25 ago 2016]; 60(1):63-64. Disponível em: http://www.inca.gov.br/rbc/n 60/v01/pdf/11-resenha-estimativa-2014incidencia-de-cancer-no-brasil.pdf.

4. Forman D, Bray F, Brewster DH, Gombe Mbalawa C, Kohler B, Piñeros M, et al. Editors. Cancer Incidence in Five Continents, Vol. X. IARC Scientific Publication No. 164. Lyon: International Agency for Research on Cancer. 2014 [acesso em: 27 ago 2016]. Available from: http://ci5.iarc.fr/CI5I-X/old/vol10/Cl5vol10.pdf.

5. Portaria №. 874, de 16 de maio de 2013 (BR) [Internet]. Institui a Política Nacional para a Prevenção e Controle do Câncer na Rede de Atenção à Saúde das Pessoas com Doenças Crônicas no âmbito do Sistema Único de Saúde (SUS). Diário Oficial da União. 17 de maio de 2013 [acesso em: 12 nov 2015]. Disponível em:

http://bvsms.saude.gov.br/bvs/saudelegis/gm/2013/prt0874_16_05 2013.html.

6. Ministério da Saúde. Controle dos cânceres do colo do útero e da mama [Internet] Brasília: Ministério da Saúde; 2013 [acesso em: 12 nov 2015]. Disponível em: http://bvsms.saude.gov.br/bvs/publicacoes/controle_canceres colo utero_2013.pdf.

7. Conselho Nacional de Secretários de Saúde. A Atenção Primária e as Redes de Atenção à Saúde [Internet] Brasília: CONASS; 2015 [acesso em: 25 ago 2016]. Disponível em: http://www.conass.org.br/biblioteca/pdf/A-Atencao- 
Primaria-e-as-Redes-de-Atencao-a-Saude.pdf.

8. Viacava F, Ugá MAD, Porto S, Laguardia J, Moreira RS. Avaliação de desempenho de saúde de sistemas: um modelo para análise. Ciênc. Saúde Colet [Internet]. 2012 [acesso em: 12 nov 2015];17(4):921-934. Disponível em:

http://dx.doi.org/10.1590/S1413-81232012000400014.

9. Barros AF, Uemura G, Macedo JLS. Atraso no diagnóstico e tratamento do câncer de mama e estratégias para a sua redução. Femina [Internet]. 2012 [acesso em: 27 ago 2016]; 40(1):31-36. Disponível em:

http://www.febrasgo.org.br/site/wp-content/uploads/2013/05/Femina-v40n1_31-36.pdf.

10. Paiva CJK, Cesse EAP. Aspectos Relacionados ao Atraso no Diagnóstico e tratamento

do Câncer de Mama em uma Unidade Hospitalar de Pernambuco. Rev Bras Cancerologia [Internet]. 2015 [acesso em: 27 ago 2016]; 61(1):23-30. Disponível em:

http://www1.inca.gov.br/rbc/n_61/v01/pdf/05-artigo-aspectos-relacionados-ao-atraso-no-diagnostico-e-tratamentodo-cancer-de-mama-em-uma-unidade-hospitalar-de-pernambuco.pdf.

11. Gonzaga CMR, Freitas-Junior R, Curado MP, Sousa ALL, Souza-Neto JA, Souza MR. Temporal trends in female breast cancer mortality in Brazil and correlations with social inequalities: ecological time-series study. BMC Public Health [Internet]. 2015 [acesso em: 27 ago 2016];15:96. Disponível em: http://dx.doi.org/10.1186/s12889-015-14457.

12. Portaria №. 876 de 16 de maio de 2013 (BR) [Internet]. Dispõe sobre a aplicação da Lei no 12.732, de 22 de novembro de 2012, que versa a respeito do primeiro tratamento do paciente com neoplasia maligna comprovada, no âmbito do Sistema Único de Saúde (SUS). Diário Oficial da União. 17 de maio de 2013 [acesso em: 12 nov 2015]. Disponível em: http://bvsms.saude.gov.br/bvs/saudelegis/gm/2013/prt0876_16_05_2013.html.

13. Oshiro ML, Bergmann A, Silva RG, Costa KC, Travaim IEB, Silva GB, et al. Câncer de Mama Avançado como Evento Sentinela para Avaliação do Programa de Detecção Precoce do Câncer de Mama no Centro-Oeste do Brasil. Rev Bras Cancerologia. [internet]. 2014 [acesso em: 25 ago 2016]; 60(1):15-23. Disponível em:

http://www.inca.gov.br/rbc/n_60/v01/pdf/04-artigo-cancer-de-mama-avancado-como-evento-sentinela-para-

avaliacao-do-programa-de-deteccao-precoce-do-cancer-de-mama-no-centro-oeste-do-brasil.pdf.

14. Coleman MP, Quaresma M, Berrino F, Lutz JM, De Angelis $R$, Capocaccia $R$, et al. Cancer survival in five continents: a worldwide population-based study (CONCORD). Lancet Oncol [Internet]. 2008 [Access in 2015 Nov 11] 9(8):730-56. Available from: http://dx.doi.org/10.1016/S1470-2045(08)70179-7.

15. Sanchez RM, Ciconelli RM. Conceitos de acesso à saúde. Rev Panam Salud Publica [Internet]. 2012 [Acesso em: 11 nov 2015] 31(3):260-268. Disponível em: http://dx.doi.org/10.1590/S1020-49892012000300012.

16. Secretaria Municipal de Saúde. Plano Municipal de Saúde: gestão 2014-2017. [Internet]. Goiânia: Secretaria Municipal de Saúde; 2014. [acesso em: 12 nov 2015]. Disponível em:

http://www.saude.goiania.go.gov.br/docs/divulgacao/PMS-Goiania-2014 a 2017.pdf.

17. Ministério da Saúde (BR). Departamento de Informação e Informática do SUS. Informações de saúde. Indicadores e dados básicos (IDB) 2012. Indicadores de cobertura. Proporção da população feminina de 50 a 69 anos que refere ter realizado a última mamografia nos últimos 2 anos, na região Centro-Oeste, no período de 2008. Brasília (DF), DATASUS, 2012 [acesso em: 2015 nov 12]. Disponível em:

http://tabnet.datasus.gov.br/cgi/tabnet.exe?idb2012/f2301.def.

18. Pan American Health Organization/World Health Organization (PAHO/WHO). Health systems and services profile. Monitoring and Analysis of Health Systems Change/Reform. Brasilia(DF); 2008 [Access in 2015 Nov 11] Available from:http://www2.paho.org/hq/dmdocuments/2010/Health_System_Profile-Brazil_2008.pdf.

19. Porto SM, Uga MAD, Moreira RS. An analysis of use of the health services by financing system: Brazil 1998-2008. Ciênc. Saúde Colet [Internet]. 2011 [Access in 2015 Nov 11] 16(9):3795-3806. Available from:

http://dx.doi.org/10.1590/S1413-81232011001000015.

20. Caleffi M, Ribeiro RA, Filho DLD, Ashton-Prolla P, Bedin Junior AJ, Skonieski GP, et al. A model to optimize public health care and downstage breast cancer in limited-resource populations in southern Brazil. Porto Alegre Breast Health Intervention Cohort. BMC Public Health. 2009 [access in 2015 Nov 10] 9(83):1-8. Available from: http://dx.doi:10.1186/1471-2458-9-83.

21. Corrêa RS. et al. Estimativas da Cobertura mamográfica no Estado de Goiás, Brasil. Cad. Saúde Pública [Internet]. 2011 [acesso em: 05 nov 2015]; 27(9):1757-1767. Disponível em: http://dx.doi.org/10.1590/S0102$311 \times 2011000900009$. 